\title{
Heat-Dependent Hairpin Melting Drives TRPV1 Opening
}

\author{
Guangyu Wang*
}

Department of Physiology and Membrane Biology, University of California School of Medicine, Davis, CA, USA

Department of Drug Research and Development, Institute of Biophysical Medico-chemistry, Reno, NV, USA

* Author to whom correspondence should be addressed; E-mail: gary.wang10@gmail.com

\begin{abstract}
The capsaicin receptor TRPV1 can be activated by heat and thus serves as a thermometer in a primary afferent sensory neuron for noxious heat detection. However, the underlying molecular mechanism is unclear. Here, a hairpin topological structural model, together with graph theory, was developed to examine a role of temperature-dependent hairpin melting in controlling noncovalent interactions along the heat-evoked gating pathway of TRPV1. The results showed that heat-dependent hairpin melting rearranges non-covalent interactions, releases the resident lipid, and induces TRPV1 gating. A larger hairpin in the outer pore initiates a temperature threshold as a heat starter for channel opening while some smaller hairpins in the S4-S5 linker and the outer pore stabilize the heat efficacy and avoid heat denaturation as a heat fuse. The heat-induced global gating rearrangement may be responsible for the high heat sensitivity. This hairpin model may provide a broad structural basis for the thermo-gated ion channels. (150 words)
\end{abstract}

Keywords: cooperative gating | graph theory | hairpin melting | ion channel $\mid$ non-covalent interaction | temperature threshold 


\section{INTRODUCTION}

Temperature is important in everyday life. Transient receptor potential (TRP) vanilloid-1 (TRPV1) is well-known as a noxious heat sensor in a primary afferent neuron of the dorsal root and the trigeminal ganglia. Its activation threshold is $\sim 42^{\circ} \mathrm{C}$ above a normal human body temperature $37^{\circ} \mathrm{C}^{[1]}$ Its activation allows sodium or calcium to be permeable to the ion channel pore, monitoring any noxious heat signal and regulating the human body temperature. ${ }^{[2-4]}$ In addition to the physical stimuli, capsaicin can also activate TRPV1, producing hot sensation. Since its activation by either physical or chemical stimuli is reversible, non-covalent interactions in TRPV1 may be involved. However, it is unclear if both physical and chemical stimuli share the same pathway to gate TRPV1, and it is also unknown what controls those non-covalent interactions during channel gating. On one hand, cold can decrease the capsaicin-evoked activity of TRPV1. [5] Thus, the same gating pathway seems to be involved. On the other hand, residues sensitive to both physical and chemical stimuli are different in TRPV1, suggesting their different gating pathways. ${ }^{[6-9]}$

Recently, a DNA hairpin thermal sensor with a 10-20 bases long poly-A loop has been created to monitor a change in a biological microenvironment. ${ }^{[10]}$ The hairpin melting temperature depends on the loop size and the plasticity, the number of H-bonded base pairs in the stem, and the ionic strength in an aqueous solution. The smaller and less flexible the hairpin loop, or the shorter the duplexed stem, or the more the H-bonds at the ends, or the higher the ionic strength in the solution, then the higher the hairpin melting temperature (Figure 1). Clearly, a short stem favors sharp hairpin melting rather than gradual melting. In any way, its melting temperatures are in a threshold range of thermosensitive TRPV channels. Its melting curve is also similar to the temperature-dependent gating of the thermosensitive TRPV channels. ${ }^{[1,6,10-16]}$ In this regard, it is fitting to ask if the thermosensitive TRPV channels work as a hairpin to control the melting temperature as their thresholds for channel gating. In this study, the cryo-electron microscopy (cryo-EM) structural data of rat TRPV1 (rTRPV1) with or without lipid-bound below and above the temperature threshold were employed to examine if TRPV1 can form the smallest hairpin topological structures to control the non-covalent interactions, how their melting temperatures control TRPV1's activation threshold, thermal sensitivity or temperature coefficient $\left(\mathrm{Q}_{10}\right)$ and heat efficacy during channel gating, and what is the role of phosphatidylinositol (PI) lipid in the heat activation (Figure 1). The gating pathways of TRPV1 initiated by both physical and chemical 
stimuli were also compared and discussed. The results are consistent with a notion that the lipidindependent hairpin melting temperatures can control at least two thermostable states for the activation threshold and the heat efficacy. The heat-induced global conformational changes along the gating pathway may determine the high thermal sensitivity. The smaller hairpins along the gating pathway may be necessary to protect TRPV1 from heat denaturation as a heat fuse. This new hairpin topological structural model may provide a broad guide for intra-molecular noncovalent interactions governing the thermo-gated ion channels.

\section{RESULTS}

\subsection{The Non-covalent Interactions Form the Primary Hairpin Topological Structures along the Gating Pathway of TRPV1 at Low Temperature}

TRPV1 is a homotetramer, with each monomer consisting of six transmembrane helices. The first four segments (S1-S4) form a voltage-sensor-like domain (VSLD) while the last two (S5 and S6), together with the intervening loop and the pore helix and the extracellular cap-like pore turret between S5 and S6, are folded as a pore domain. The pore domain is surrounded by the VSLD in a domain-swapped arrangement. The cation conductance pathway centers in the pore domain. A hydrophobic constriction around I697 forms a lower S6 gate while a short selectivity filter $\left({ }^{643} \mathrm{GMGD}^{646}\right)$ between S5 and S6 (S5-P-S6) in rTRPV1 acts as an upper gate. The S4-S5 linker, which is a short amphipathic helix and almost parallel to the cytoplasmic membrane, couples VSLD with the pore domain for channel gating. The gating rearrangements in both upper and lower gates can also be coupled together to drive channel opening. Thus, the overall structure of the TRPV1 channel resembles that of the voltage-gated ion channel. ${ }^{[17-18]}$ However, the TRPV1 channel has a conserved TRP domain, which runs parallel to the inner leaflet of the membrane by virtue of a sharp bend after S6, not only linking the S6 gate crossing bundle but also interacting with the S4-S5 linker and the pre-S1 domain in favor of allosteric coupling between different gating domains. In addition, the characteristic ankyrin repeats within the cytoplasmic $\mathrm{N}$ terminus can tether cytoplasmic $\mathrm{N}$ - and C-terminal domains for channel assembly. ${ }^{[18-21]}$

The cryo-EM structures of rTRPV1 at different temperatures are now available to examine

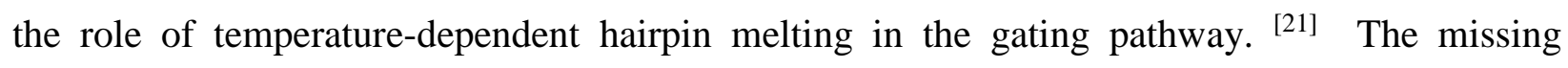
information of the cap-like pore turret in the wide outer pore can be integrated with the full-length cryo-EM structure of squirrel TRPV1 (sqTRPV1) because both rTRPV1 and sqTRPV1 share most highly conserved residues in their primary sequences (Figure S1). Figure 2 shows that non- 
covalent interactions play a critical role in forming hairpins to link different gating domains together for an allosteric activity coupling (Figure 2).

First, several pairwise residues are available for hairpin-forming salt bridges. They include R409 in the pre-S1 domain and D509 in the S2-S3 linker, K425 in the pre-S1 domain and E709 in the TRP domain, R491 or R499 on S2 and D707 in the TRP domain, K571 or R575 in the S4-S5 linker and E692 in the TRP domain, R579 on S5 and E684 on S6, and K603 or R617 in the pore turret and D654 or E651 in the pore loop, respectively (Figure 2).

Second, $\pi-\pi$ interactions between aromatic side rings along the gating pathway also bring about hairpins (Figure 2). In the VSLD and its interface with the pre-S1 domain, there are four groups with edge-face $\pi-\pi$ interactions between different helix segments. The first includes Y441 on S1, F516 on S3 and Y554 or Y555 on S4; the second has F434 and Y435 on S1, W426 on preS1 and F559 in the S4-S5 linker; the third group members are Y530 on S3 and Y537 on S4; the fourth contains F522 on S3 and F543 on S4. In contrast, four groups in the pore domain have faceface or edge-face $\pi-\pi$ interactions between several pairwise aromatic residues. They are Y666 on S6 and F591 on S5 or F640 on the pore helix, Y627 on the pore helix and F649 on the pore loop, both Y653 and F659 on the pore loop, F580 and Y584 on S5 (Figure 2).

Third, H-bonds between different segments also contribute the formation of hairpins. These H-bonding pairs are Y401 or Q423 on pre-S1 and D707 or R701 in the TRP domain, respectively, S512 on S3 and R557 on S4, Q519 on S3 and N551 on S4, Q560 in the S4-S5 linker and K694 in the TRP domain, D576 on S5 and T685 on S6, Y584 on S5 and T641 on the pore helix or Y666 on S6 (Figure 2).

Fourth, the resident PI in the capsaicin pocket also bridges some residues together to produce hairpins via $\mathrm{H}$ - bonds or electrostatic attractions. For example, R557 on S4 and R409 on pre-S1 or Q700 in the TRP domain or E570 in the S4-S5 linker, Q700 in the TRP domain and E570 in the S4-S5 linker (Figure 2).

Finally, the sulfur from M644 in the selectivity filter and the hydroxyl from Y671 on S6 between two neighbor subunits can bind a cation $\mathrm{Na}^{+}$in the permeation pathway to form an intersubunit metal bridge. Taken together, there are several hairpin groups with the smallest loop sizes to control non-covalent interactions along the gating pathway: the first group with 0 to 36 -residue loops in the outer pore; the second group with 3 to 6-residue loops between S5 and S6; the third 
group with 0 to 8-residue loops between the S4-S5 linker and the TRP domain; and the forth group with 0 to 10-residue loops in the VSLD and its interface with the pre-S domain (Figure 2).

\subsection{The Role of the Resident PI Lipid in the Formation of Hairpins}

The resident PI is occupided in the vanilloid pocket. ${ }^{[22]}$ When capsain competes off the PI lipid at a low temperature, this vanilloid compound does not bridge any residue in rTRPV1 to cause new hairpins under the given nanodisc condition. ${ }^{[21]}$ However, the release of the PI lipid disconnects several non-covalent interactions along the gating pathway and thus decreases hairpin numbers from the second hairpin group to the fourth. The disconnected salt-bridging pairs include R491-D707, E692-K571/R575 and E684-R579. The broken PI-bridging pairs cover R557Q700/E570/R409 and Q700-E570. In the meantime, new H-bonds between Q700 in the TRP domain and R557 on S4 or E570 in the S4-S5 linker are generated, together with an E684-K688 salt bridge and an Q691-N695 H-bond. As a result, the smallest hairpin sizes are altered as 3-14, 3-12 and 0-17 residues in the loops in the second, third and fourth hairpin group, respectively. However, the first hairpin group in the outer pore is not affected and still has 36 and 21 residues in the primary and secondary largest loops (Figures 2-3, Figure S2).

\subsection{The Removal of Metal Ions between the Selectivity Filters by a Warm Temperature Releases the Dynamic Selectivity Filter}

The cryo-EM structures of lipid-free rTRPV1 below and above the temperature threshold $\left(42^{\circ} \mathrm{C}\right)$ are available for examination of the hairpin topological structural model (Figure 1). When the temperature raises from $4{ }^{\circ} \mathrm{C}$ to $25^{\circ} \mathrm{C}$, the inter-subunit M644-M644 and Y671-Y671 metal bridges are broken and the resultant hairpins with a 36-residue loop are first open and the dynamic selectivity filter is released. The metal removal in the upper gate does not open the channel but initiates several changes along the gating pathway (Figure 3, Figure S2). First, the R575-E692 salt bridge is restored, the E684-K688 salt bridge is broken but E692 also forms a salt bridge with nearby K688. Therefore, a small hairpin with 3-residues in the loop is generated in the second hairpin group. Second, the R499-D707 salt bridge is disrupted while the R491-D707 salt bridge is restored and a new R499-D509 salt bridge is present. Thus, hairpins with 3-10 and 9-15 residues in the loops in the third and fourth hairpin groups are produced, respectively. This global conformational change demonstrates that the pore domain is coupled to the VSLD via the interaction between the S4-S5 linker and the TRP domain (Figure 3, Figure S2). 


\subsection{A Larger Hairpin in the Outer Pore Acts as a Heat Starter to Initiate a Temperature Threshold for Channel Opening}

When the temperature continues to increase above the threshold $42^{\circ} \mathrm{C}$, the hairpin with a 21 residue loop from Y627 to F649 in the first hairpin group is first open in the intermediate state (Figure 3, Figure S2). In the meantime, the E648-K656 salt bridge is also broken. However, D601 in the pore turret forms an H-bond network with N628 in the same pore turret and N652 in the pore loop. F640 in the pore helix also produces an edge-face $\pi-\pi$ attractive interaction with F649 in the pore loop. The remaining smallest hairpin sizes are in a range from 0 to 16 residues in the loops to control the non-covalent interactions in the outer pore.

When this conformational change in the first hairpin group extends to the second and third ones, the E692-R575/K688 salt bridge and the Q560-K694 H-bond are disrupted while Q691 and E570 H-bond with K694 and R557, respectively. In this case, the related hairpins with 3 to 10residue loops are open but hairpins with 0 to 16-residue loops are formed (Figure 3, Figure S2).

When this conformational change extends to the fourth hairpin group, multiple non-covalent interactions are broken. They include K425-E709 and R491-D707 and D509-R409 salt bridges, Q423-R701 and S512-R557 H-bonds, and the Y441-Y555 $\pi-\pi$ interaction. In the meanwhile, the W426-R701 cation- $\pi$ interaction, the F434-Y554 $\pi-\pi$ interaction, and the Y554-T704 H-bond are formed. The remaining smallest hairpins are in a size range from 0 to 10 residues in loops to control the relevant non-covalent interactions from the second hairpin group to the fourth (Figure 3, Figure S2).

Taken together, the larger hairpin in the outer pore acts as a heat starter to initiate a temperature threshold for channel opening. The resultant hairpins with the size larger than 16 residues in the loops are open (Figure 3, Figure S2).

\subsection{Smaller Hairpins in Both the Outer Pore and the S4-S5 Linker Stabilize the Heat Efficacy and Function as a Heat Fuse to Prevent Heat Denaturation}

When the temperature is much higher than the threshold, K603-D654 and R617-E651 salt bridges and F640-Y666/F649 $\pi-\pi$ interactions are broken in the first hairpin group. In the meantime, the N628-N652 H-bond and the F649-F659 $\pi-\pi$ interaction emerge. The resultant dilation of the dynamic selectivity filter opens the upper gate. The remaining smallest hairpins with 0 to 14-residue loops control the non-covalent interactions in the first hairpin group. In the second hairpin group, the $\pi-\pi$ interaction shifts from F580-Y584 to Y584-F587 (Figure 4). 
Once the upper gate is opened by heat, the coupled lower gate at I679 on S6 is also open. As a result, Y401-D707 and E570-Q700/R557 and Q691-K694/N695 H-bonds, the W426-R701 cation- $\pi$ interaction, F434-Y554 and Y441-F516 $\pi-\pi$ interactions are disrupted in the third and fourth hairpin groups. In the meantime, W426-R701 and S512-R557 H-bonds, the Y441-Y555 $\pi-\pi$ interaction, and R557-E570 and K688-E692 salt bridges are formed. In this case, the resultant smallest hairpin sizes are ranged from 0 to 16 residues in the loops in the third and fourth hairpin groups. Although the edge-face $\pi-\pi$ interactions are kept between F516 and Y554/Y555, the edgeface $\pi-\pi$ interaction between Y554 and Y555 is broken due to the reoriented side chain of Y555. Once the R557-E570 salt bridge is formed in the third hairpin group, the uplift of the S4-S5 linker stabilizes the lower open gate (Figure 4).

In both closed and open states, some non-covalent interactions are shared. They include the $\pi-\pi$ interactions between W426 and F434/F435, Y441 and Y555, F516 and Y554/Y555, F522 and F543, F530 and Y537, F591 and Y666, and Y653 and F659, the H-bonds between R557 and Q700/S512, D576 and T685, and Y584 and Y666/T641. Therefore, these non-covalent interactions and the related hairpins may form a stable backbone anchor system for thermosensitive TRPV1 and also serve as a heat fuse to prevent heat denaturation. ${ }^{[18-19,21,23]}$ On the other hand, the R557-E570 salt bridge, the N652-D601/N628 H-bond network, and the F649-F659 edge-face $\pi-\pi$ interaction are present in the open state. Accordingly, these emerging non-covalent interactions and relevant small hairpins from the outer pore to the S4-S5 linker may play a critical role in stabilizing the heat efficacy (Figures 2-4).

\section{DISCUSSION}

TRPV1 is designed and created as one of biological thermometers in the thermostable system to detect noxious heat and to protect an animal body from heat damage. ${ }^{[2-4]}$ To this end, it should meet several engineering requirements. First, it should be an integral membrane protein with a relative high physicochemical stability or heat capacity to avoid thermal denaturation. Second, it should be an ion channel to directly couple a temperature signal to an electronical signal reporter for a readout by a primary afferent sensory neuron. Third, it should have a high Q10 with a steep response to a small change in a surround predetermined temperature. Fourth, it should have a heat switch with two temperature threshold limits for two gating states to protect itself from heat denaturation, one for activation and the other for inactivation. In the biological and chemical 
world, a hairpin topological structure may be an ideal tool to control a high Q10 and different temperature thresholds.

The DNA hairpin with 10-20 bases in the loop has been designed as a temperature switch, a thermometer and an ionic detector with Q10 as $1.32 .{ }^{[10]}$ It has oligonucleotides with complementary nucleotide sequences at the ends to form a duplex region as the hairpin stem upon intra-strand hybridization, and the nucleotides in between as the hairpin loop. When a temperature increases above a threshold, the DNA hairpin will melt and thus becomes single stranded. Its melting temperature sharply depends on the H-bonded base pairs in the stem, the size and flexibility of the loop and the ionic strength in a solution. Generally, a stable stem or a small and rigid loop or a high salt concentration in the solution allow the DNA hairpin to have a high threshold to sense and to respond to highly specific and small changes in an environmental temperature. Usually, the melting temperature can be increased more than 20 degrees if the loop length is decreased from 20 to 10 A's or the H-bonded G-C base-pairs raises from 2 to 4 . However, a change in the loop sequence has no significant effect on the melting temperature. ${ }^{[10]}$

Previous study of a cyclic $\beta$-hairpin peptide of the general sequence XRTVXVdPGOXITQX demonstrated that although a hydrophobic cluster is typically necessary for stabilizing a $\beta$-hairpin, ${ }^{[24-25]}$ the aromatic residues stabilize the hairpin more than the salt bridges at a non-hydrogen-bonded internal and terminal position. The aromatic pairs enthalpically prefer a favorable edge-face $\pi-\pi$ attractive interaction, whereas the folding of hairpins with crossstrand salt bridge is more entropically favorable than enthalpically favorable. Thus, aromatic $\pi-\pi$ attractive interactions play a pivotal role in forming a tightly-folded hairpin and thus increasing enthalpy and decreasing entropy. ${ }^{[26]}$

In the case of TRPV1, the channel activity is controlled by the coupled gating rearrangements in a hydrophobic constriction at the cytoplasmic S6 gate and the dynamic selectivity filter formed by S5-P-S6. ${ }^{[19,21,27]}$ The presence of the inter-subunit metal bridge between M644 and M644 or Y671 and Y671 may lock the channel in the closed state. ${ }^{[9,21]}$ However, its disruption cannot open the channel (Figures 2-3). Other non-covalent interactions can generate multiple hairpin topological structures along the gating pathway as the basis for its thermometer design with a high Q10 value. They include salt bridges and aromatic side chain $\pi-\pi$ interactions and the cation- $\pi$ interaction and $\mathrm{H}$-bonds. Both small and large hairpins with the upper and lower temperature limits are also available to couple with channel gating. In a physiological 
salt concentration range required for the heat sensitivity, ${ }^{[9]}$ rTRPV1 uses the edge-face $\pi-\pi$ interaction between Y627 and F649 to engineer a larger hairpin with a 21-residue loop in the outer pore to detect a noxious heat $\left(>42^{\circ} \mathrm{C}\right)$. This hairpin is coupled to the dynamic selectivity filer to control the upper gate of the channel. Therefore, its opening or melting above a temperature threshold allows the dilation of the dynamic selectivity filer for channel opening. Both R617-E651 and K603-D654 salt bridges, together with the edge-face $\pi-\pi$ interaction between F640 and Y666 may directly weaken the plasticity of the upper gate and thus increase the threshold. ${ }^{[27]}$ The small hairpin from Y653 to F659 may ensure the basic skeleton of the dynamic selectivity filter. That is why TRPV1 with double-knot toxin (DkTx) bound in the outer pore cannot be further activated by heat. ${ }^{[9]}$ Once the first hairpin loop from Y627 to F649 is open, the globle conformational change can be extended with the heat waves from the outer pore to other gating domains and result in a series of hairpin rearrangments. Finally, the R557-E570 salt bridge in the S4-S5 linker, together with the N652-D601/N628 H-bond network and the F649-F659 edge-face $\pi-\pi$ interaction in the outer pore, stabilizes the open state in both upper and lower gates and thus the high heat efficacy (Figures 2-4, Figure S2). ${ }^{[23]}$ Supporting this proposal, the N628D mutation in the outer pore dramatically enhances the heat sensitivity (Q10 86) while the N628L mutation in the outer pore and the M572A mutation in the S4-S5 linker greatly decrease the heat sensitivity Q $10(\sim 1.7-$ 2.6). ${ }^{[21]}$

Regarding the change in a free energy during heat activation of TRPV1, the opening of multiple larger hairpins increase entropy as a result of the disruption of most of the salt bridges along the gating pathway. However, the formation of new smaller hairpins, together with conserved aromatic edge-face $\pi-\pi$ attractive interactions and H-bonds, increase enthalpy to secure the heat capacity. Thus, a small decrease in the free energy is enough for intra-molecular gating rearrangements to activate TRPV1 and to meet its engineering requirement for noxious heat detection. On the other hand, the short hairpin stem with a non-covalent interaction, together with the coupled gating rearrangements of hairpins along the gating pathway, may allow the product of multiple related smaller Q10 values upon hairpin opening and closure to be responsible for the high thermal sensitivity or the large Q10 (Figures 3-4, Figure S2).

Since the resident PI lipid in the capsaicin pocket does not increase the hairpin size beyond the larger hairpin with the 21-residue loop in the outer pore, the PI lipid may not alter the temperature threshold. Once the larger hairpin opens upon the heat stimuli above the threshold, 
the resultant conformational change may be extended from the first hairpin group to the fourth and thus release the resident PI lipid in the vanilloid pocket to form the stimulatory R557-E570 salt bridge. ${ }^{[22]}$ Therefore, the release of the resident PI lipid is required to stabilize the heat efficacy. Finally, the removal of the resident PI lipid by capsaicin mainly disrupts the relevant PI or salt bridges but the channel is still closed at $25^{\circ} \mathrm{C} .{ }^{[21]}$ In this regard, the effect of the resident PI in the vanilloid pocket on the heat sensitivity or Q10 may be weak (Figures 2-3, Figure S2).

Previous studies showed that the outer pore or N628, T633, N652, Y653, F640, F649 at the S5-P-S6 are sensitive to heat. ${ }^{[7,28-33]}$ The N628K or the N652T or Y653T or combined mutation can raise the temperature threshold for TRPV1 opening. ${ }^{[7]}$ In this case, it is possible that the N652T or Y653T mutation in rTRPV1 may disrupt the face-face $\pi-\pi$ interaction between Y653 and F659 while the N628K mutation may allow the formation of a putative salt bridge with D601. In any way, K603-D654 and R617-E651 salt bridges may be enhanced but the flexibility of the dynamic selectivity filter may be decreased and thus the temperature threshold is increased. ${ }^{\text {[7] }}$ In support of this notion, when the turret deletion or replacement with glycine between G603 and N626 disrupts the equivalent K604-D655 or R618-E652 salt bridge in mTRPV1, the temperature threshold is lowered to a different extent. However, the disruption of both salt bridges stops channel opening (Figures 2-4, Figures S1-2). ${ }^{[8]}$ In contrast, $\mathrm{Mg}^{2+}$ may bind to N652 and D601 and N628 in mTRPV1 and thus increase the plasticity of the dynamic selectivity filter for the decreased activation threshold in mTRPV1. ${ }^{[34]}$

Both capsaicin and heat can activate rTRPV1 eliciting hot sensation. However, they are different in several aspects. First, capsaicin potency and efficacy are based on the same binding site above the S4-S5 linker and between S3/S4 and S5/S6. Either the R557-E570 salt bridge or the vanilloid bridge via H-bonds between the side chains of T551 on S4 and Y511 in the S3-S4 linker or E570 in the S4-S5 linker can prime the capsaicin affinity and efficacy by uplifting the S4-S5 linker. ${ }^{[20-21,35]}$ However, the heat threshold and efficacy in lipid-bound rTRPV1 may be separated. The larger hairpin with the 21-residue loop from Y627 to F649 in the outer pore may be responsible for the heat threshold while the R557-E570 salt bridge in the S4-S5 linker, together with the N652-D601/N628 H-bond network and the F649-F659 $\pi-\pi$ interaction in the outer pore, may stabilize the heat efficacy (Figures 2-4). Second, capsaicin starts a gating rearrangement from the VSLD and ends at the outer pore while heat may initiate a global gating rearrangement from the outer pore to the VSLD, the S4-S5 linker, the TRP domain, pre-S1 domain and C-terminal 
domain. ${ }^{[19-21,27]}$ Third, capsaicin competes off the resident lipid via the anchor-stereoselective vanilloid bridge in the capsaicin pocket. ${ }^{[35]}$ In contrast, heat may open the hairpin loops around the resident PI lipid and then release it.

The prior study suggested that F435 on S1, F516 on S3, Y545 and Y555 and R557 may form a putative menthol pocket in hTRPV1. The side chain of R557 may anchor the H-bonded homochiral menthol dimer and thus disrupt the stimulatory R557-E570 salt bridge. ${ }^{[36]}$ Since menthol only partly supresses the capsaicin-elicited hTRPV1 current, ${ }^{[37]}$ capsaicin can alternatively stimulate channel opening by bridging E570 or Y511 with T550 via side chain Hbonds in the absence of the R557-E570 salt bridge. ${ }^{[20-21,35,38]}$ On the other hand, menthol only partly stop the heat activation of TRPV1 without changing the temperature threshold. [37] Accordingly, the release of the resident PI lipid by menthol may not alter the threshold for heat activation of TRPV1. However, once heat releases the resident PI lipid, the disruption of the stimulatory R557-E570 salt bridge by menthol may not completely suppress the heat efficacy. ${ }^{[21,}$ 37-38] In contrast, niacin may H-bond with the side chains of capsaicin-sensitive S512 and R557 and E570 to release the resident PI lipid in favor of the formation of the stimulaotry R557-E570 salt bridge to decrease the activation energy for mTRPV1 opening. ${ }^{[35]}$ That's why niacin can decrease the temperature threshold of mTRPV1 activation by heat from $38.92^{\circ} \mathrm{C}$ to $24.88^{\circ} \mathrm{C}$. ${ }^{[39]}$ In fact, when the capsaicin-sensitive residues S512 and E570 in TRPV1 have been replaced with Phe and Gln residues in other TRPV channels, respectively, niacin can not activate TRPV2 and TRPV3 and TRPV4. ${ }^{[40]}$

In addition to TRPV1, other TRPV channels than TRPV5-6 are also in warm and hot sensation. Their temperature thresholds for activation are TRPV1 $\left(>42{ }^{\circ} \mathrm{C}\right)$, TRPV2 $\left(>52{ }^{\circ} \mathrm{C}\right)$, TRPV3 (>30-39 $\left.{ }^{\circ} \mathrm{C}\right)$, TRPV4 $\left(>25-35^{\circ} \mathrm{C}\right) .{ }^{[1,11-16]}$ Although they have different temperature thresholds, sequence alignment of the TRPV family indicates that some hairpin-forming residues are highly conserved to a different extent for the thermo-sensation among human TRPV1-4 (hTRPV1-4) and sqTRPV1 and rTRPV1 and mTRPV1 but not in hTRPV5-6 (Figure S1). For example, the equivalent $\pi-\pi$ interactions between F516 and Y554/Y555, Y441 and F516, Y530 and Y537, W426 and Y435, Y584 and F587, the equivalent R499-D509 salt bridge, and the equivalent Y584-T641/Y666 H-bond network. Therefore, these shared non-covalent interactions and related hairpins may provide a common structural basis for warm to heat activation of TRPV1- 
4 channels. ${ }^{[41]}$ In contrast, even if some hairpins are present along the gating pathway in thermoinsensitive TRPV5-6, their sizes may be small enough to protect them from heat activation.

Non-covalent interactions have been reported to regulate the gating pathway of ion channels at room temperatures. ${ }^{[35-36,42-50]}$ Here, this study further demonstrates that the smallest hairpin sizes in the molecular systemic network may control thermostablity of those non-covalent interactions in thermo-gated TRPV1 channels. Further investigation is necessary to prove this notion in other thermosensitive biological macromolecules.

\section{CLOSING REMARK}

The hairpin topological structures can be used to design a thermosensitive biological thermometer with a high Q10 value. In addition to ionic strength in the physiological range, the hairpin size and the loop plasticity and non-covalent interactions such as salt bridges and aromatic side chain $\pi-\pi$ interactions and cation- $\pi$ interactions and $\mathrm{H}$-bonds and metal bridges can be used to tune different melting temperatures as thresholds for the detection of distinct small environmental temperature changes. Both larger and smaller hairpins are necessary to control the lower and upper temperature limits. Their opening and closure should be reversible and fast enough to have a very large effect on channel gating, which can be easily coupled to an electrical readout in the primary afferent sensor neuron. In this regard, this study may provide a broad structural basis for how thermosensitive TRP channels work as a thermometer.

\section{MATERIALS AND METHODS}

In this in silicon study, the hairpin topological structural aspects of TRPV1 channels below and above the temperature threshold were investigated and analyzed with graph theory as the thermometer basis for noxious heat detection. The in silico protomer models were based on the cryo-EM structural data of closed rTRPV1 with PI-bound at $4{ }^{\circ} \mathrm{C}$ (PDB ID, 7LP9), capsaicin-bound at $4^{\circ} \mathrm{C}$ (PDB ID, 7LPA) and $25^{\circ} \mathrm{C}$ (PDB ID, 7LPB) and $48^{\circ} \mathrm{C}$ (PDB ID, 7LPD), open rTRPV1 with capsaicin-bound at $48^{\circ} \mathrm{C}$ (PDB ID, 7LPE), ${ }^{[21]}$ and the closed apo sqTRPV1 (PDB ID, 7LQY). [20] UCSF Chimera was used for in silicon analyses. All the potential non-covalent interactions such as salt-bridges and $\pi-\pi$ interactions and cation- $\pi$ interactions and H-bonds and metal bridges along the gating pathway in TRPV1 were included to test their potential roles in forming the smallest hairpins, controlling the temperature threshold and the temperature sensitivity Q10, and stablizing the heat afficacy. The smallest hairpin sizes to control the intensity of non-covalent interactrions were calculated using graph theory. The different gating pathways for activation of 
TRPV1 by heat and capsaicin were also compared. Q10 was used to characterize the temperaturedependent responses as calculated using the following equation:

$$
\mathrm{Q}_{10}=(\mathrm{R} 2 / \mathrm{R} 1)^{10 /(\mathrm{T} 2-\mathrm{T} 1)}
$$

Conventions and Abbreviations: cryo-EM, cryo-electron microscopy; DkTx, double-knot toxin or vanillotoxin; PI, phosphatidylinositol; TRP, transient receptor potential; TRPV1, TRP vanilloid-1; hTRPVi, human TRPVi (i=1; 2, 3, 4, 5, 6); mTRPV1, mouse TRPV1; rTRPV1, rat TRPV1; sqTRPV1, squirrel TRPV1; VSLD, voltage-sensor-like domain

\section{Acknowledgements}

The author's own studies cited in this article were supported by NIDDK Grant (DK45880 to D.C.D.) and Cystic Fibrosis Foundation grant (DAWSON0210) and NIDDK grant (2R56DK056796-10) and American Heart Association (AHA) Grant (10SDG4120011 to GW).

Conflict of Interest: The author declares no conflict of interest.

Data Availability Section: This study includes no data deposited in external repositories 


\section{References}

1. M. J. Caterina, M. A. Schumacher, M. Tominaga, T. A. Rosen, J. D. Levine, D. Julius. The capsaicin receptor: a heat-activated ion channel in the pain pathway. Nature 1997, 389, 816.

2. J. B. Davis, J. Gray, M. J. Gunthorpe, J. P. Hatcher, P. T. Davey, P. Overend, M. H. Harries, J. Latcham, C. Clapham, K. Atkinson, S. A. Hughes, K. Rance, E. Grau, A. J. Harper, P. L. Pugh, D. C. Rogers, S. Bingham, A. Randall, S. A. Sheardown. Vanilloid receptor-1 is essential for inflammatory thermal hyperalgesia. Nature 2000, 405,183.

3. N. R Gavva; J. J. S. Treanor, A. Garami, L. Fang, S. Surapaneni, A. Akrami, F. Alvarez, A. Bak, M. Darling, A. Gore, G. R. Jang, J. P. Kesslak, L.Ni, M. H. Norman, G. Palluconi, M. J. Rose, M. Salfi, E. Tan, A. A. Romanovsky, C. Banfield, G. Davar. Pharmacological blockade of the vanilloid receptor TRPV1 elicits marked hyperthermia in humans. Pain 2008, 136, 202.

4. I. Vandewauw, K. De Clercq, M. Mulier, K. Held, S. Pinto, N. V. Ranst, A. Segal, T. Voet, R. Vennekens, K. Zimmermann, J. Vriens, T. Voets. A TRP channel trio mediates acute noxious heat sensing. Nature 2018, 555, 662.

5. M. K. Chung, S. Wang. Cold suppresses agonist-induced activation of TRPV1. J Dent Res 2011, 90, 1098.

6. S. E. Jordt, D. Julius. Molecular basis for species-specific sensitivity to "hot" chili peppers. Cell 2002, 108, 421.

7. J. Grandl, S. E. Kim, V. Uzzell, B. Bursulaya, M. Petrus, M. Bandell, A. Patapoutian. Temperature-induced opening of TRPV1 ion channel is stabilized by the pore domain. Nat Neurosci. 2010, 13, 708.

8. Y. Cui, F. Yang, X. Cao, V. Y. Yarovoy, K.W. Wang, J. Zheng. Selective disruption of high sensitivity heat activation but not capsaicin activation of TRPV1 channels by pore turret mutations. J. Gen. Physiol. 2012, 139, 273.

9. A. Jara-Oseguera, C. Bae, K. J. Swartz. An external sodium ion binding site controls allosteric gating in TRPV1 channels. Elife 2016, 5.

10. A. T. Jonstrup, J. Fredsøe, A. H. Andersen. DNA Hairpins as Temperature Switches, Thermometers and Ionic Detectors. Sensors 2013, 13, 5937. 
11. M. J. Caterina, T. A. Rosen, M. Tominaga, A. J Brake, D. Julius. A capsaicin-receptor homologue with a high threshold for noxious heat. Nature 1999, 398,436.

12. M. J. Caterina, A. Leffler, A. B. Malmberg, W. J. Martin, J. Trafton, K. R. Petersen-Zeitz, M. Koltzenburg, A. I. Basbaum, D. Julius. Impaired nociception and pain sensation in mice lacking the capsaicin receptor. Science 2000, 288, 306.

13. A. M. Peier, A. J. Reeve, D. A. Andersson, A. Moqrich, T. J. Earley, A. C. Hergarden, G. M. Story, S. Colley, J. B. Hogenesch, P. McIntyre, S. Bevan, A. Patapoutian. A heatsensitive TRP channel expressed in keratinocytes. Science. 2002, 296, 2046.

14. G. D. Smith, M. J. Gunthorpe, R. E. Kelsell, P. D. Hayes, P. Reilly, P. Facer, J. E. Wright, J. C. Jerman, J-P. Walhin, L. Ooi, J. Egerton, K. J. Charles, D. Smart, A. D. Randall, P. Anand, J. B. Davis. TRPV3 is a temperature-sensitive vanilloid receptor-like protein. Nature 2002, 418, 186.

15. H. Xu, I. S. Ramsey, S. A. Kotecha, M. M. Moran, J. A. Chong, D. Lawson, P. Ge, J. Lilly, I. Silos-Santiago, Y. Xie, P. S. DiStefano, R. Curtis, D. E. Clapham. TRPV3 is a calciumpermeable temperature-sensitive cation channel. Nature 2002, 418, 181.

16. M. K. Chung, H. Lee, M. J. Caterina. Warm temperatures activate TRPV4 in mouse 308 keratinocytes. J. Biol. Chem. 2003, 278, 32037.

17. S. B. Long, E. B. Campbell, R. Mackinnon. Crystal structure of a mammalian voltagedependent Shaker family $\mathrm{K}^{+}$channel. Science 2005, 309, 897.

18. M. Liao, E. Cao, D. Julius, Y. Cheng. Structure of the TRPV1 ion channel determined by electron cryo-microscopy. Nature 2013, 504,107.

19. E. Cao, M. Liao, Y. Cheng, D. Julius. TRPV1 structures in distinct conformations reveal activation mechanisms. Nature 2003, 504, 113.

20. K. D. Nadezhdin, A. Neuberger, Y. A. Nikolaev, L. A. Murphy, E. O. Gracheva, S. N. Bagriantsev, A. I. Sobolevsky. Extracellular cap domain is an essential component of the TRPV1 gating mechanism. Nat Commun. 2021, 12, 2154.

21. D. H. Kwon, F. Zhang, Y. Suo, J. Bouvette, M. J. Borgnia, S.-Y. Lee, Heat-dependent opening of TRPV1 in the presence of capsaicin. Nat Struct Mol Biol. 2021, 28, 554.

22. Y. Gao, E. Cao, D. Julius, Y. Cheng. TRPV1 structures in nanodiscs reveal mechanisms of ligand and lipid action. Nature 2016, 534, 347. 
23. S. Boukalova, L. Marsakova, J. Teisinger, V. Vlachova. Conserved residues within the putative S4-S5 region serve distinct functions among thermosensitive vanilloid transient receptor potential (TRPV) channels. J. Biol. Chem. 2010, 285, 41455.

24. A. J. Maynard, G. J. Sharman, M. S. Searle. Origin of $\beta$-hairpin stability in solution: Structural and thermodynamic analysis of the folding of model peptide supports hydrophobic stabilization in water. J. Am. Chem. Soc. 1998, 120, 1996.

25. J. F. Espinosa, S. H. Gellman. A designed $\beta$-hairpin containing a natural hydrophobic cluster. Angew Chem. Int. Edit. 2000, 39, 2330.

26. S. E. Kiehna, M. L. Waters. Sequence dependence of $\beta$-hairpin structure: Comparison of a salt bridge and an aromatic interaction. Protein Science 2003, 12, 2657.

27. K. Zhang, D. Julius, Y. Cheng. Structural snapshots of TRPV1 reveal mechanism of polymodal functionality. Cell 2021, 184, 1.

28. S. Ryu, B. Liu, J. Yao, Q. Fu, F. Qin. Uncoupling proton activation of vanilloid receptor TRPV1. J. Neurosci 2007, 27, 12797.

29. B. R. Myers, C. J. Bohlen, D. Julius. A yeast genetic screen reveals a critical role for the pore helix domain in TRP channel gating. Neuron 2008, 58, 362.

30. M. Papakosta, C. Dalle, A. Haythornthwaite, L. Cao, E. B. Stevens, G. Burgess, R. Russell, P. J. Cox, S. C. Phillips, C. Grimm. The chimeric approach reveals that differences in the TRPV1 pore domain determine species-specific sensitivity to block of heat activation. $J$ Biol Chem. 2011, 286, 39663.

31. S. E. Kim, A. Patapoutian, J. Grandl. Single residues in the outer pore of TRPV1 and TRPV3 have temperature-dependent conformations. PLoS One 2013, 8, e59593.

32. C Bae, C. Anselmi, J. Kalia, A. Jara-Oseguera, C. D. Schwieters, D. Krepkiy, C. W. Lee, E.-H. Kim, J. Kim, J. D. Faraldo-Gómez, K. J. Swartz. Structural insights into the mechanism of activation of the TRPV1 channel by a membrane-bound tarantula toxin. Elife, 2016, 5 .

33. F Zhang, A. Jara-Oseguera, T.-H. Chang, C. Bae, S. M Hanson, K. J. Swartz. Heat activation is intrinsic to the pore domain of TRPV1. Proc. Natl. Acad. Sci. USA. 2018, 115, E317.

34. X. Cao, L. Ma, F. Yang, K. W. Wang, J. Zheng. Divalent cations potentiate TRPV1 channel by lowering the heat activation threshold. J Gen Physiol. 2014, 143, 75. 
35. G. Wang. Lipid-dependent sequential allosteric activation of heat-sensing TRPV1 channels by anchor-stereoselective "hot" vanilloid compounds and analogs. Biochem. Biophys. Rep. 2000, 28, 101109.

36. G Wang. Ligand-stereoselective allosteric activation of cold-sensing TRPM 8 channels by an H-bonded homochiral menthol dimer with head-to-head or head-to-tail. Chirality 2021, $33,783$.

37. M Takaishi, K. Uchida, Y. Suzuki, H. Matsui, T. Shimada, F. Fujita, M. Tominaga. Reciprocal effects of capsaicin and menthol on thermosensation through regulated activities of TRPV1 and TRPM8. J Physiol Sci. 2016, 66, 143.

38. T. H. D Nguyen, S. G. Itoh, H. Okumura, M. Tominaga. Structural basis for promiscuous action of monoterpenes on TRP channels. Commun Biol. 2021, 4, 293.

39. L. Ma, B. H. Lee, R. Mao, A. Cai, Y. Jia, H. Clifton, S. Schaefer, L. Xu, J. Zheng. Nicotinic Acid Activates the Capsaicin Receptor TRPV1 Potential Mechanism for Cutaneous Flushing. Arterioscler Thromb Vasc Biol. 2014, 34, 1272.

40. L. Ma, B. H. Lee, H. Clifton, S. Schaefer, J. Zheng. Nicotinic Acid is a Common Regulator of Heat-Sensing TRPV1-4 Ion Channels. Sci. Rep. 2015, 5, 8906.

41. M. Kim, N. J. Sisco, J. K. Hilton, C. M. Montano, M. A. Castro, B. R. Cherry, M. Levitus, W. D. V. Horn. Evidence that the TRPV1 S1-S4 membrane domain contributes to thermosensing. Nat Commun. 2020, 11, 4169.

42. G Wang. State-dependent regulation of cystic fibrosis transmembrane conductance regulator (CFTR) gating by a high affinity $\mathrm{Fe}^{3+}$ bridge between the regulatory domain and cytoplasmic loop 3. J Biol Chem 2010, 285, 40438.

43. W. Wang, J. Wu, K. Bernard, G. Li, G. Wang, W. Bevensee, K. L. Kirk. ATP-independent CFTR channel gating and allosteric modulation by phosphorylation. Proc Natl Acad Sci USA, 2010, 107, 3888.

44. G. Wang. The inhibition mechanism of non-phosphorylated Ser768 in the regulatory domain of cystic fibrosis transmembrane conductance regulator. J Biol Chem. 2011, 286, 2171.

45. G. Wang, D. D. Duan. Regulation of activation and processing of the cystic fibrosis transmembrane conductance regulator (CFTR) by a complex electrostatic interaction between the regulatory domain and cytoplasmic Loop 3. J Biol Chem 2012, 287, 40484. 
46. G. Wang. Interplay between inhibitory ferric and stimulatory curcumin regulates phosphorylation-dependent human cystic fibrosis transmembrane conductance regulator and $\Delta \mathrm{F} 508$ activity. Biochemistry 2015, 54, 1558.

47. G. Wang. Molecular Basis for Fe(III)-Independent Curcumin Potentiation of Cystic Fibrosis Transmembrane Conductance Regulator Activity. Biochemistry 2015, 54, 2828.

48. G. Wang, R. Linsley, Y. Norimatsu. External $\mathrm{Zn}^{2+}$ binding to cysteine-substituted cystic fibrosis transmembrane conductance regulator constructs regulates channel gating and curcumin potentiation. FEBS J 2016, 283, 2458.

49. G. Wang. Mechanistic insight into the heme-independent interplay between iron and cartbon monoxide in CFTR and Slo1 BKca channels. Metallomics 2017, 9, 634.

50. G. Wang. Removal of the Fe(III) site promotes activation of the human cystic fibrosis transmembrane conductance regulator by high-affinity $\mathrm{Zn}(\mathrm{II})$ binding. Metallomics 2018, $10,240$. 


\section{Figure legends}

Figure 1 The hairpin test model for examining the gating pathway of heat-sensing TRPV1 channels. Non-covalent interactions such as salt bridges and aromatic side chain $\pi-\pi$ interactions and H-bonds in the TRPV1 channel are proposed to form hairpins with different loop sizes. The large and small loops have low and high melting temperatures as primary thresholds for hairpin opening, respectively. The physical and chemical stimuli including ionic strength on the hairpin loop can regulate the loop plasticity for different melting temperatures. Only hairpin opening and closure along the gating pathway may determine the thresholds for TRPV1 gating.

Figure 2. The non-covalent interactions (A) and the hairpins map (B) of lipid-bound rTRPV1 along the gating pathway in the closed state at $4^{\circ} \mathrm{C}$. A, The cryo-EM structures of apo sqTRPV1 (PDB ID, 7LQY) and apo rTRPV1 (PDB ID, 7LP9) in the presence of the resident PI lipid were used for the integral model. ${ }^{[20-21]}$ Salt and metal and PI bridges and H-bonds and $\pi-\pi$ interactions enhance the intra- and inter-domain interactions along the gating pathway. The residues are colored in red for a positive charge or blue for a negative charge in salt bridges or H-bonds, orange for the uncharged residues in H-bonds, green for $\pi-\pi$ interactions and dark blue for the metal bridge. The PI lipid is marked in grey. The aromatic pairs for $\pi-\pi$ interactions are clustered in the pore domain and the VSLD and the interface between the pre-S1 domain and the VSLD. B, The pore domain, the S4-S5 linker, the TRP domain, the VSLD and the pre-S1 domain are indicated. Salt or PI bridges, $\pi-\pi$ interactions, the metal bridge and H-bonds are marked in purple, green, dark blue and orange, respectively. The smallest hairpin loop sizes required to control the non-covalent interactions were calculated using graph theory and labeled.

Figure 3. The hairpins maps of lipid-free rTRPV1 along the gating pathway at $4^{\circ} \mathrm{C}(\mathrm{A})$ and $25^{\circ} \mathrm{C}$ (B) and $48^{\circ} \mathrm{C}(\mathrm{C})$. Non-covalent interactions along the gating pathway were based on the cryoEM structure of lipid-free rTRPV1 at $4^{\circ} \mathrm{C}$ (PDB ID, 7LPA) (A), $25^{\circ} \mathrm{C}$ (PDB ID, 7LPB) (B) and $48^{\circ} \mathrm{C}$ (PDB ID, 7LPD) (C) and Figure 2A. ${ }^{[20-21]}$ The pore domain, the S4-S5 linker, the TRP domain, the VSLD and the pre-S1 domain are indicated. Salt bridges, $\pi-\pi$ interactions, the cation$\pi$ interaction and H-bonds are marked in purple, green, dark green and orange, respectively. The 
smallest hairpin loop sizes required to control the non-covalent interactions were calculated using graph theory and labeled.

Figure 4. The non-covalent interactions (A) and the hairpins map (B) of the lipid-free rTRPV1 along the gating pathway in the open state at $48^{\circ} \mathrm{C}$. A, The cryo-EM structure of capsaicin-bound rTRPV1 at $48^{\circ} \mathrm{C}$ (PDB ID, 7LPE) was used to evaluate the non-covalent interactions for channel opening. ${ }^{[21]}$ Salt bridges and H-bonds and $\pi-\pi$ interactions enhance the intra- and inter-domain interactions along the gating pathway. The residues are colored in red for a positive charge or blue for a negative charge in salt bridges or H-bonds, orange for the uncharged residues in H-bonds, and green for $\pi-\pi$ interactions. The aromatic pairs for $\pi-\pi$ interactions are clustered in the pore domain and the VSLD and the interface between the pre-S1 domain and the VSLD. B, The pore doamin, the S4-S5 linker, the TRP domain, the VSLD and the pre-S1 domain are indicated. Salt bridges, $\pi-\pi$ interactions and $\mathrm{H}$-bonds are marked in purple, green and orange, respectively. The smallest hairpin loop sizes required to control the non-covalent interactions were calculated using graph theory and labeled. 


\section{Figures}

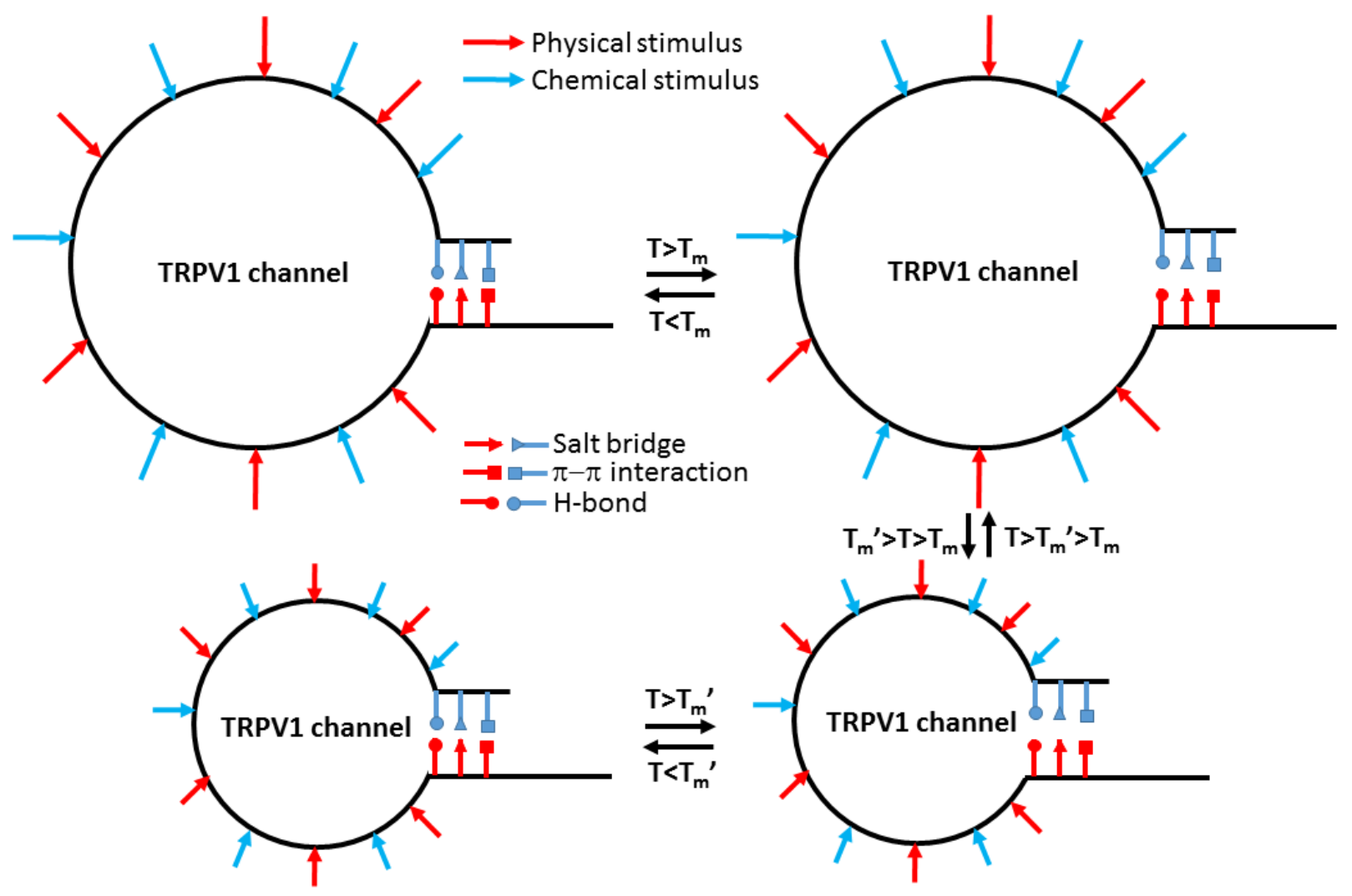

Figure 1 
A

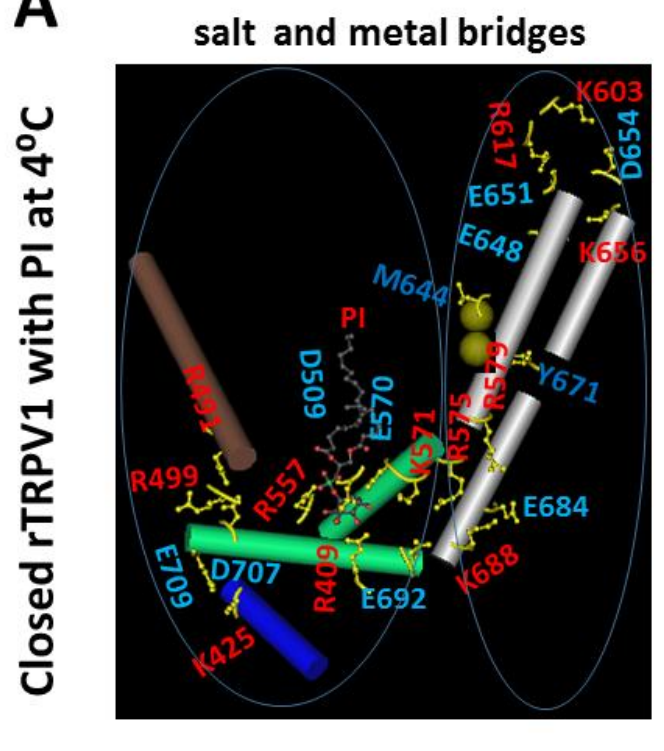

B

\section{Closed rTRPV1 with PI at $4^{\circ} \mathrm{C}$}

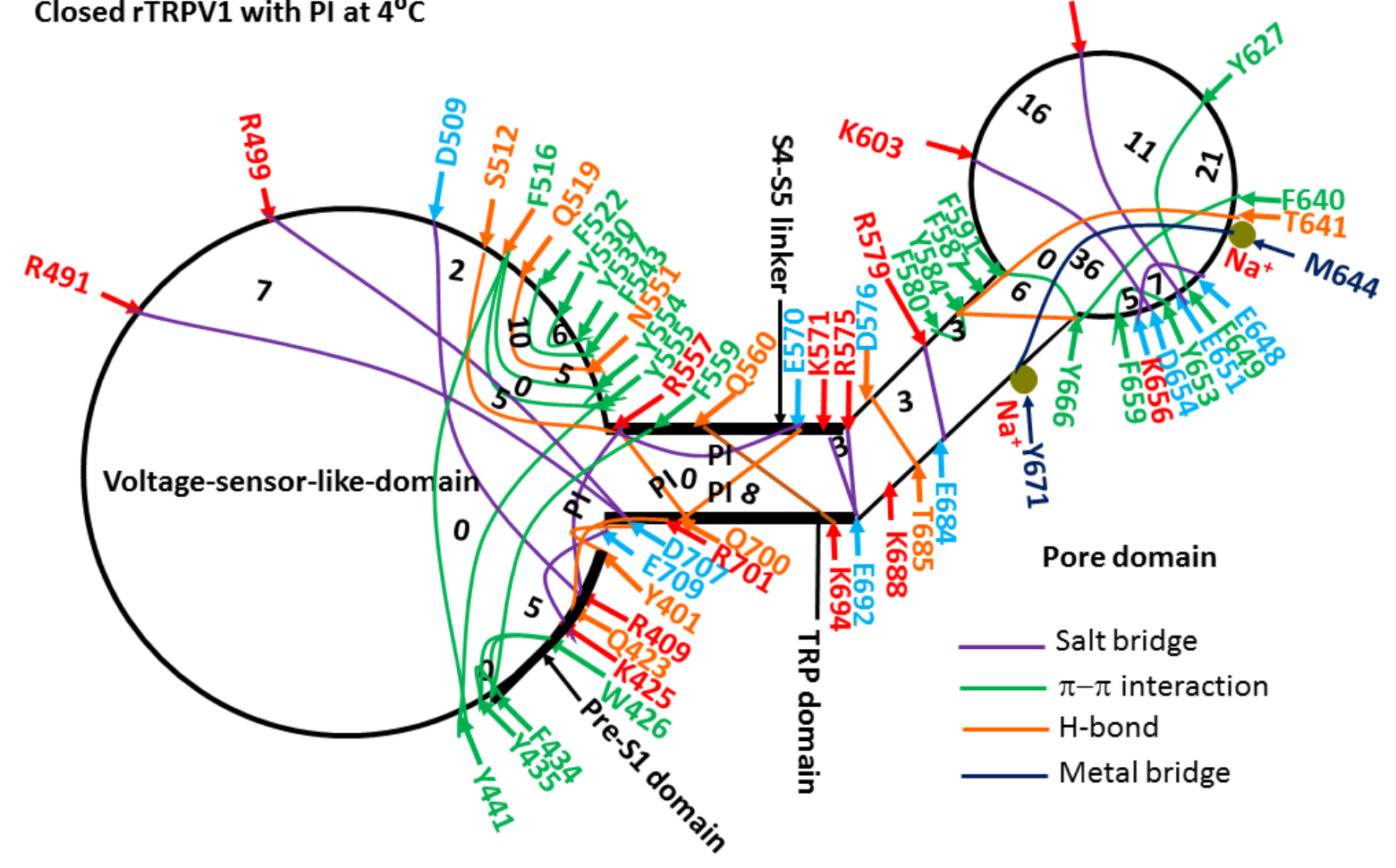

Figure 2 
bioRxiv preprint doi: https://doi.org/10.1101/2022.01.02.474701; this version posted January 10,2022 . The copyright holder for this preprint (which was not certified by peer review) is the author/funder. All rights reserved. No reuse allowed without permission.

A

Closed rTRPV1 without PI at $4^{\circ} \mathrm{C}$

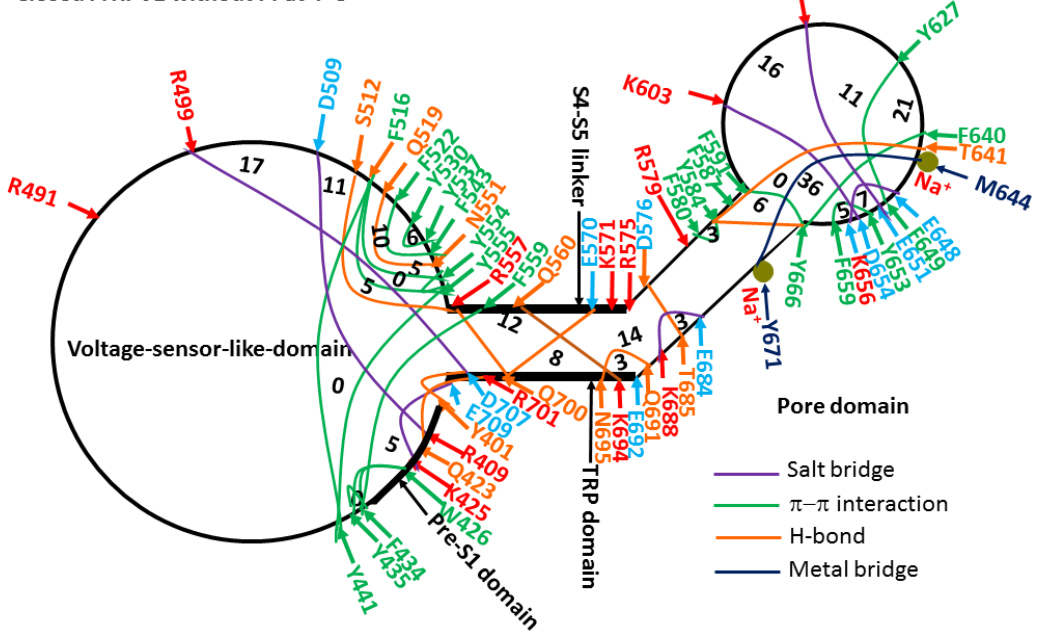

B

Closed rTRPV1 without PI at $25^{\circ} \mathrm{C}$

C

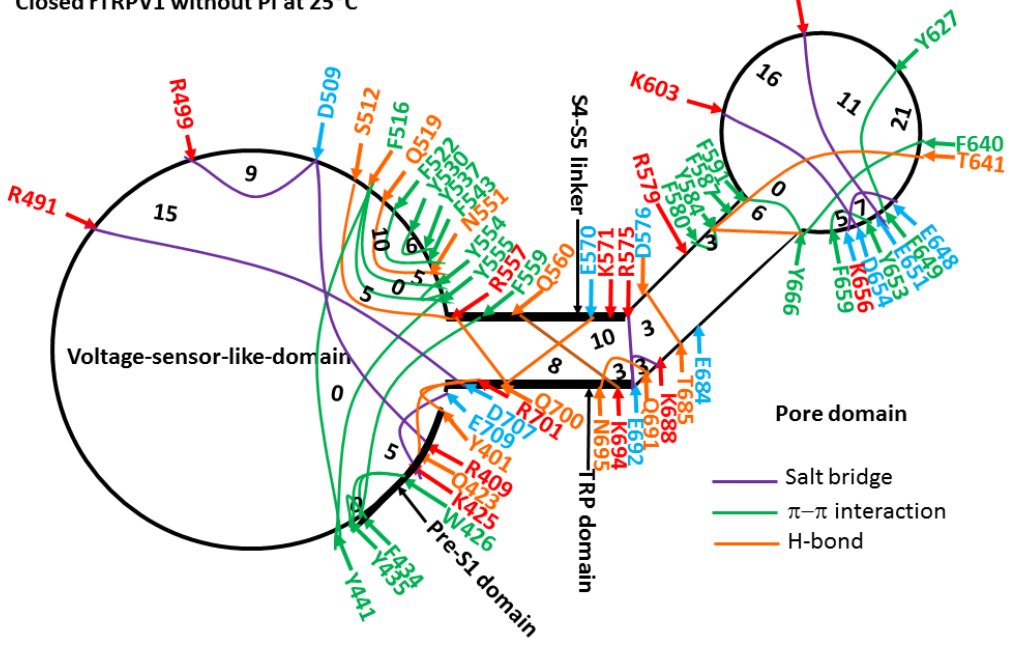

Intermediate rTRPV1 without PI at $48^{\circ} \mathrm{C}$

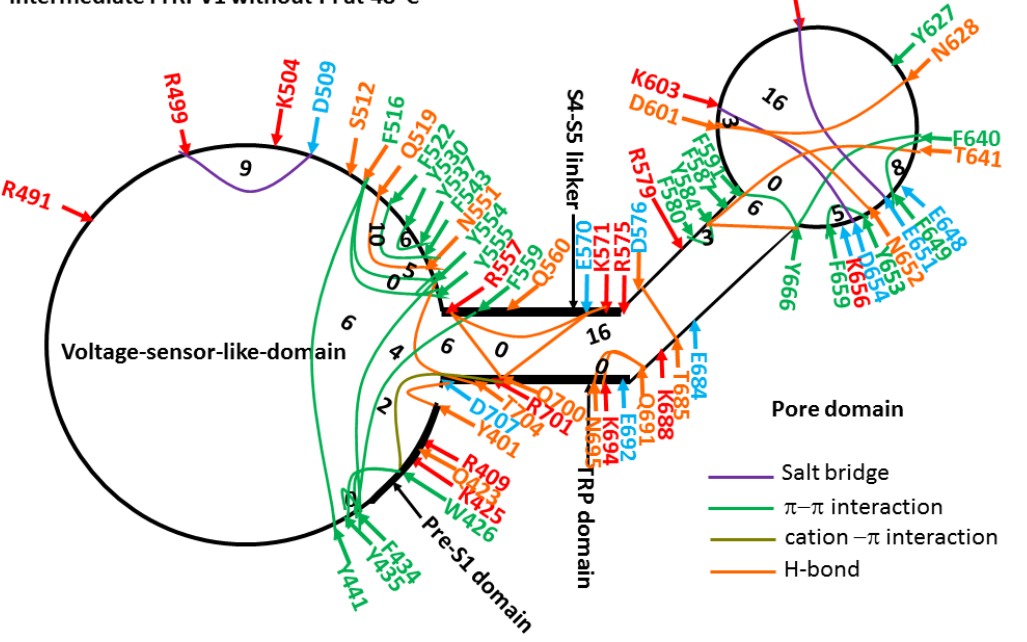

Figure 3 
A

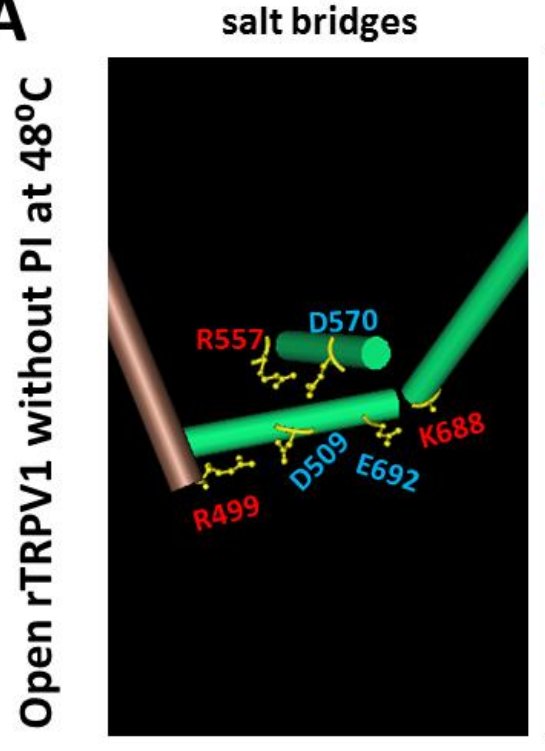

B

Open rTRPV1 without PI at $48^{\circ} \mathrm{C}$
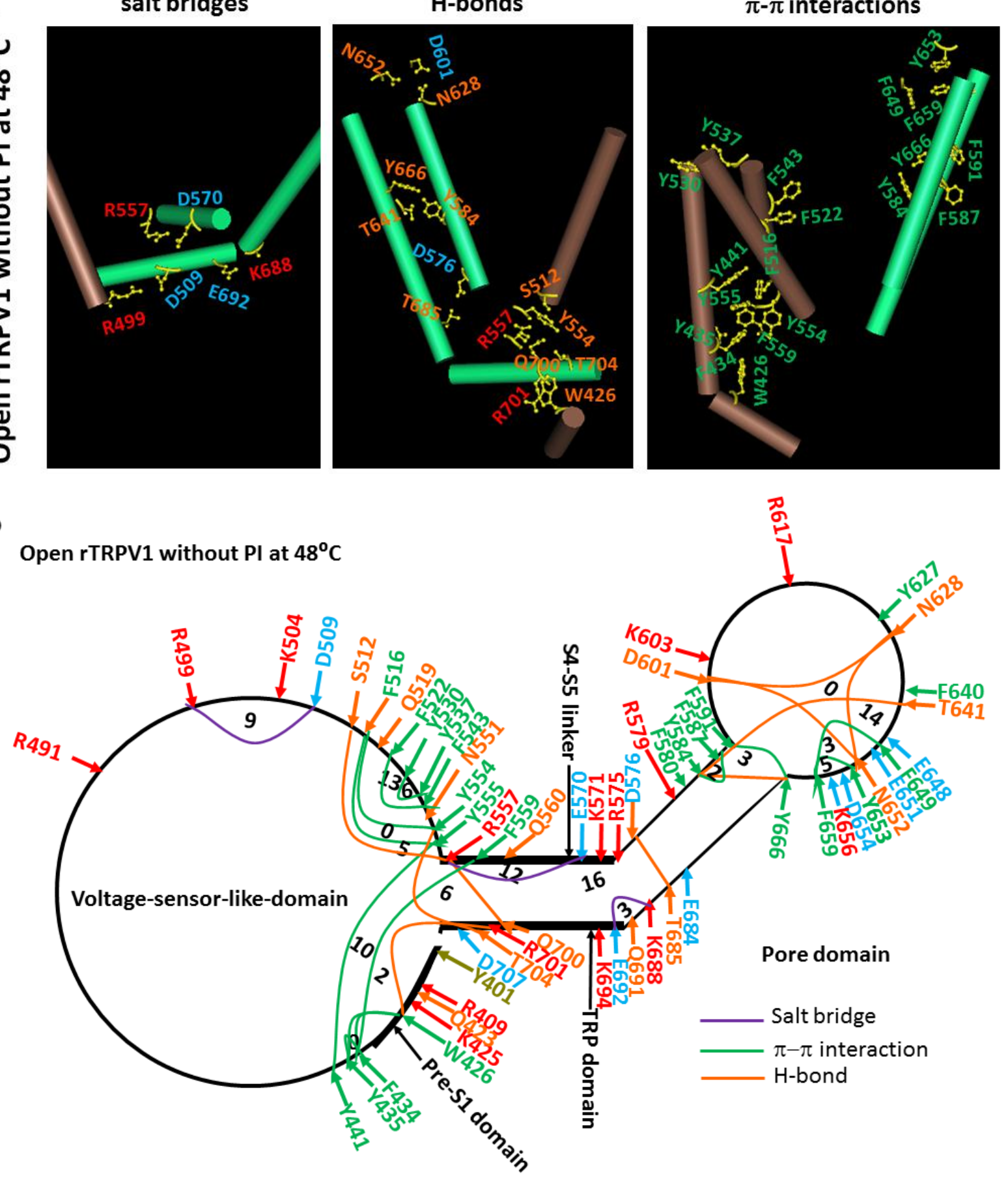

Figure 4 